\title{
High-Temperature Membrane Reactor for Catalytic Gas-Solid Reactions
}

\author{
H. J. Sloot, C. A. Smolders, W. P. M. van Swaaij, and G. F. Versteeg \\ Dept. of Chemical Engineering, Twente University of Technology, 7500 AE Enschede, The Netherlands
}

A mathematical model, based on the dusty-gas model extended with surface diffusion, is presented that describes mass transport owing to molecular diffusion and viscous flow, as well as an instantaneous reversible reaction inside a membrane reactor. The reactants are fed to opposite sides of the membrane, considering masstransfer resistances in the gas phase outside the membrane. The Claus reaction is chosen as a model reaction to study this membrane reactor.

The model is used to validate a previously presented simplified model. The simplified model predicts correct molar fluxes when it is very dilute and can therefore be considered a pseudo-binary system. Occurrence of a maximum or a minimum in the pressure profile inside the membrane, in the absence of an overall pressure difference over the membrane, depends not only on the stoichiometry of the reaction but on mobilities of the different species.

The Claus reaction is used to verify experimentally the transport model for a nonpermselective membrane reactor with a mean pore diameter of $350 \mathrm{~nm}$. At 493 $K$ and $542 \mathrm{~K}$, molar fluxes experimentally determined are 10 to $20 \%$ lower than those predicted by the transport model. Conversions measured at pressures of 220 $k P a$ and $500 \mathrm{kPa}$ demonstrate that surface diffusion occurs as a transport mechanism despite the large pore diameter of the membrane. In the presence of a pressure difference over the membrane, there is a reasonable agreement between experimentally determined molar fluxes and those calculated by the transport model.

\section{Introduction}

Many processes in the chemical industry encounter extremely rapid, often strongly exothermic, heterogeneously catalyzed gas-solid reactions. The operation of these processes is very complicated because of problems like thermal runaway, loss of selectivity, catalyst deactivation and/or destruction, and explosion phenomena, if premixed feed streams have to be used. Processes with severe constrictions on the stoichiometry of the feed of the reactants, such as the catalytic reduction of $\mathrm{NO}_{x}$ with $\mathrm{NH}_{3}$, are very difficult to operate. For these types of processes, a novel type of membrane reactor has been developed and investigated in which a (ceramic) membrane has two functions. First, it is used to keep reactants separated from each other and have them come together in a controlled way

Correspondent concerning this article should be addressed to G. F. Versteeg.

Present address of H. J. Sloot: Koninklijke/Shell Laboratorium, Amsterdam, P.O. Box 3003, 1003 AA Amsterdam, The Netherlands. instead of using the membrane to separate components and second, as (or supporting) the catalyst. We (Sloot et al., 1990) previously presented a simplified transport model for this reactor. In this article, a fundamentally more correct model based on the dusty-gas model (Mason and Malinauskas, 1983) is presented which also includes surface diffusion as an additional transport mechanism.

In this reactor, reactants are fed at opposite sides of a porous membrane that contains a catalyst for a heterogeneously catalyzed reaction between the reactants. The reactants diffuse from either side into the membrane and therefore a pressure difference over the membrane, in principle, is not necessary, in contrast to the usual applications of membranes. Inside the membrane, a reaction between the reactants occurs and the products obtained diffuse out of the membrane to both sides. If the rate of the chemical reaction is faster than the diffusion rates of the reactants, a small reaction zone or, in the case of 
an instantaneous irreversible reaction, a reaction plane inside the membrane results. The location of this reaction zone will be such that the molar fluxes of the reactants are in stoichiometric ratio. If for some reason the concentration of one of the reactants increases, this will evolve in a higher molar flux of this reactant into the membrane. At the reaction zone, an excess of this reactant will be present and therefore this component will penetrate further into the membrane before the reaction takes place. This results in a shift of the reaction zone toward the side of the reactant whose molar flux is below stoichiometry. Due to this shift, the distance from the membrane interface to the reaction zone decreases for this reactant resulting in an increase of its molar flux and vice versa for the other reactant. The new location of the reaction zone will be such that the molar fluxes of both reactants are in stoichiometric ratio again.

Due to the fast reaction inside the membrane, slip of the reactants to the opposite side of the membrane is not possible when the reaction is irreversible. Because of the stoichiometric molar fluxes and the impossibility of slip of the reactants to the opposite side of the membrane, this reactor could be very suitable for chemical processes requiring strict stoichiometric feed rates of reactants.

A mathematical model is developed to study this membrane reactor and to determine the influence of reversibility of the reaction and of a pressure difference over the membrane that can occur in practice. This model describes mass transport owing to molecular diffusion and viscous flow through the membrane in combination with an instantaneous, reversible reaction inside the membrane. As a fundamental transport model in porous media, the so-called dusty-gas model (Mason and Malinauskas, 1983) extended with surface diffusion, has been used. A simplified transport model describing transport combined with an instantaneous reversible reaction inside a membrane was presented earlier (Sloot et al., 1990). This simplified model uses Fick's diffusion equations for describing the diffusion of all components as well as effective binary diffusion coefficients. The use of Fick's diffusion equation in describing multicomponent diffusion, however, is valid only in very diluted, pseudo-binary systems. This model, based on a fundamentally more correct and more widely valid transport model, can, therefore, be used to validate the simplified model. A number of new effects are also predicted by the present model.

To investigate the properties and performance of the membrane reactor theoretically and experimentally, the Claus reaction was selected as a model reaction (Mark et al., 1983):

$$
2 \mathrm{H}_{2} \mathrm{~S}+\mathrm{SO}_{2} \leftrightarrow \frac{3}{8} \mathrm{~S}_{8}+2 \mathrm{H}_{2} \mathrm{O}
$$

because this reaction can be regarded as a good example of a heterogeneously catalyzed gas-solid reaction with the restriction of strict stoichiometric feed rates of $\mathrm{H}_{2} \mathrm{~S}$ and $\mathrm{SO}_{2}$ to avoid emission of one of the reactants. Note, however, that the Claus reaction was selected as a model system and must not be regarded as the optimally suitable process to be performed in the membrane reactor. Since the Claus reaction is an equilibrium reaction, the presence of substantial amounts of water in the feed gas has a very pronounced effect on removing the sulfur efficiently from the process.

\section{Model Description}

The flux model presented here describes mass transport in a membrane in combination with an instantaneous, reversible reaction inside the membrane with reactants coming from different sides of the membrane. Transport is described in the stationary state in the direction perpendicular to a membrane with a flat sheet geometry, reflecting the experimental setup used. To simulate a practical application using porous membranes this flux model must be modified to cylindrical coordinates and be incorporated into a reactor model taking the operation mode (co- or countercurrent) into account.

The instantaneous, reversible reaction is:

$$
\nu_{A} A+\nu_{B} B \stackrel{\kappa_{\text {eq }}}{\curvearrowleft} \nu_{C} C+\nu_{D} D
$$

The basic variables to be calculated as a function of the position in the membrane are the pressure $P$ and the mole fractions of the components $A, B, C$, and $D$, if present, of the inert components. As in the present model, one inert component is taken into account and six variables have to be calculated as a function of the position in the membrane and therefore six equations are required. The differential mass balance for a component $i$ is presented by Eq. 2 :

$$
\frac{d J_{i}}{d z}=R_{i}(z)
$$

with $R_{i}<0$ for reactants and $R_{i}>0$ for products.

And when an inert species is present:

$$
\frac{d J_{\text {inert }}}{d z}=0
$$

As the reaction rate in Eq. 2 is considered instantaneous compared to mass transport, the reaction rate $R_{A}$ is indefinite. Therefore, $R_{A}$ is eliminated by adding and subtracting the terms in Eq. 2 resulting in four independent equations (Eqs. 4-7).

$$
\begin{aligned}
& \frac{1}{\nu_{A}} \frac{d J_{A}}{d z}-\frac{1}{\nu_{B}} \frac{d J_{B}}{d z}=0 \\
& \frac{1}{\nu_{A}} \frac{d J_{A}}{d z}+\frac{1}{\nu_{C}} \frac{d J_{C}}{d z}=0 \\
& \frac{1}{\nu_{A}} \frac{d J_{A}}{d z}+\frac{1}{\nu_{D}} \frac{d J_{D}}{d z}=0
\end{aligned}
$$

The fifth equation required is the equilibrium condition assumed to be valid through the entire membrane:

$$
K_{\mathrm{eq}}=\frac{x_{C}^{\nu_{C}} x_{D}^{\nu_{D}}}{x_{A}^{\nu_{A}} x_{B}^{\nu_{B}}} P^{\nu_{C}+\nu_{D}-\nu_{A}-\nu_{B}}
$$

The sum of the mole fractions must be equal to 1 , which is the sixth and final equation:

$$
\sum_{i=1}^{i=n c} x_{i}=1
$$


To solve the set of equations (Eqs. 4-9) additional equations are required specifying the molar fluxes, $J_{i}$, as a function of the driving forces. The dusty-gas model (Mason and Malinauskas, 1983), extended with surface diffusion (Sloot, 1991), is used to obtain the expressions for $J_{i}$. The total molar flux $J_{i}$ of a component $i$ through the pores of the membrane with a mean pore diameter $d_{p}$ is calculated according to Eq. 10 assuming cylindrically shaped pores:

$$
J_{i}=J_{i, \text { gas }}+\frac{4}{d_{p}} J_{i, \text { surf }} \quad i=1, \ldots, n c
$$

Equation 10 reflects the fact that transport through the gas phase in the pores of the membrane is proportional to the cross-sectional area of the pores and therefore proportional to $d_{p}^{2}$, whereas transport owing to surface diffusion is proportional to the circumstances of the pores and therefore proportional to $d_{p}$.

The molar flux of a component $i$ through the gas phase in the pores is calculated by Eq. 11 (Mason and Malinauskas, 1983), omitting thermodiffusion and external forces such as gravitation fields in fast rotating systems. The gas phase is assumed to behave as an ideal gas:

$$
\begin{aligned}
& \sum_{j=1}^{j=n c}\left(\frac{x_{j} J_{i, \text { gas }}-x_{i} J_{j, \mathrm{gas}}}{P D_{i j}}\right)+\frac{J_{i, \mathrm{gas}}}{P D_{i K}} \\
& =-\frac{x_{i}}{R T}\left(\frac{1}{P}+\frac{B_{o}}{\eta D_{i K}}\right) \frac{d P}{d z}-\frac{1}{R T} \frac{d x_{i}}{d z} \quad i=1, \ldots, n c
\end{aligned}
$$

The first term on the lefthand side of Eq. 11 represents continuum diffusion written in the Stefan Maxwell formulation of diffusion and the second term represents Knudsen diffusion. The first term on the righthand side reflects transport caused by a pressure gradient and consists of a pressure diffusion term and a viscous flow contribution. The second term on the righthand side gives the transport owing to a mole fraction gradient. The effective continuum and Knudsen diffusion coefficients used in Eq. 11 are determined by Eqs. 12.

$$
D_{i j}=\frac{\epsilon}{\tau} D_{i j}^{o} \text { and } D_{i K}=\frac{\epsilon}{\tau} D_{i K}^{o}
$$

The continuum binary diffusion coefficients $D_{i j}^{o}$ are calculated by the gas kinetic theory (Reid et al., 1977) and the Knudsen diffusion coefficients $D_{i K}^{o}$ by Eq. 13:

$$
D_{i K}^{o}=\frac{d_{p}}{3} \sqrt{\frac{8 R T}{\pi M_{i}}}
$$

Equations 11-13 contain only two variables that have to be specified; the porosity-tortuosity factor $\epsilon / \tau$ and the average pore diameter $d_{p}$. The membrane specific parameter $B_{o}$ is related to $\epsilon / \tau$ and $d_{p}$ according to Eq. 14 (see, for example, Mason and Malinauskas, 1983):

$$
B_{o}=\frac{\epsilon}{\tau} \frac{d_{p}^{2}}{32}
$$

Surface diffusion is described by Fick's law as presented by Eq. 15:

$$
J_{i, \text { suff }}=-D_{i, s} c_{s} \frac{d \theta_{i}}{d z} \quad i=1, \ldots, n c
$$

In Eq. 15, $D_{i, S}$ is the effective diffusion coefficient of surface diffusion of component $i, c_{S}$ is the concentration of adsorption sites $\left(\mathrm{mol} / \mathrm{m}^{2}\right)$, and $\theta_{i}$ is the fraction of the adsorption sites covered by component $i$. The rates of adsorption and desorption are assumed to be fast compared to the transport rate through the membrane, and therefore an instantaneous adsorption-desorption equilibrium is obtained. It is assumed that the fraction of the adsorption sites covered by component $i$, $\theta_{i}$, can be described by a Langmuir isotherm as:

$$
\theta_{i}=\frac{b_{i} x_{i} P}{1+P \sum_{j=1}^{j=n c} b_{j} x_{j}} i=1, \ldots, n c
$$

Equations 15-16 contain $n c$ effective coefficients, $D_{i S}$, $n c$ adsorption constants, $b_{i}$, and the total concentration of adsorption sites, $c_{s}$, whose values have to be specified beforehand in the calculations.

To solve Eqs. 4-16 uniquely, 12 boundary conditions are required. At the membrane interfaces the equilibrium condition (Eq. 8) must be fulfilled and also the sum of the mole fractions has to be equal to 1 (Eq. 9). The pressure at the membrane interfaces must be equal to the pressure in the bulk of the gas at that side of the membrane (Eq. 17).

$$
P_{\text {int }}=P_{\text {bulk }} \quad z=0 \text { and } z=L
$$

There are still six additional boundary conditions required.

Mass-transfer resistances outside the membrane are taken into account. Because outside of the membrane no reaction takes place and inside the membrane the reaction is assumed to be instantaneous, the molar fluxes from the bulk of gas to the membrane interface $J_{i, b-i}$ and the molar fluxes in the membrane at the interface $J_{i, \text { int }}$ do not have to be equal to each other, but are coupled by the stoichiometry of the reaction. Owing to the assumption of an instantaneous reaction, continuity of both the molar fluxes and the mole fractions at the membrane interface cannot be obeyed. As the condition of continuity of the mole fractions must always be satisfied, continuity of the molar fluxes at the membrane interfaces is not possible. Equations 18-21 represent the coupling of the molar fluxes $J_{i, b-i}$ and $J_{i, \text { int }}$ by the stoichiometry of the reaction.

$$
\begin{aligned}
& \frac{1}{v_{B}}\left(J_{B, b-i}-J_{B, \text { int }}\right)-\frac{1}{\nu_{A}}\left(J_{A, b-i}-J_{A, \text { int }}\right)=0 \\
& \\
& z=0 \text { and } z=L
\end{aligned}
$$

$$
\frac{1}{\nu_{C}}\left(J_{C, b-i}-J_{C, \text { int }}\right)+\frac{1}{\nu_{A}}\left(J_{A, b-i}-J_{A, \text { int }}\right)=0
$$$$
z=0 \text { and } z=L
$$ 


$$
\begin{aligned}
& \frac{1}{\nu_{D}}\left(J_{D, b-i}-J_{D, \text { int }}\right)+\frac{1}{\nu_{A}}\left(J_{A, b-i}-J_{A, \text { int }}\right)=0 \\
& \quad z=0 \text { and } z=L \\
& J_{\text {iner }, b-i}=J_{\text {iner }, \text { int }} z=0 \text { and } z=L
\end{aligned}
$$

There are eight mass balances at the membrane interfaces to be fulfilled and only six boundary conditions required to solve Eqs. 4-16 uniquely, and therefore two mass balances seem to be superfluous.

Equations 18-21 introduce five new variables, $J_{A, b-i}, J_{B, b-i}$, $J_{C, b-i}, J_{D, b-i}$ and $J_{\text {inert,b-i}}$ at $z=0$ and at $z=L$, and therefore ten additional equations specifying these molar fluxes from the bulk of gas to the membrane interface are required. To describe the combination of flow and diffusion outside the membrane the film model is used. To specify the molar fluxes in the stagnant gas film caused by diffusion the Stefan Maxwell diffusion Eq. 22 is applied:

$$
\sum_{j=1}^{j=n c} \frac{x_{j} J_{i, \mathrm{dif}}-x_{i} J_{j, \mathrm{dif}}}{P D_{i j}^{o}}=-\frac{1}{R T} \frac{d x_{i}}{d z} \quad i=1, \ldots, n c-1
$$

The reason for the use of the Stefan Maxwell diffusion equation, instead of Fick's law, is that these equations are fundamentally to be preferred to Fick's diffusion equation in describing multicomponent diffusion, especially for the diffusion of the inert component that is present in large amounts.

Equations 22 give the diffusion fluxes of the components relative to each other. In case of a pressure difference over the membrane, a part of the transport through the membrane is caused by viscous flow (see Eq. 11), and this flow through the membrane also should pass the stagnant gas films representing mass-transfer resistances in the gas phase outside the membrane. Since this is a flow through free space, the pressure drop in the stagnant gas film is negligible. The combination of flow with diffusion is represented by Eq. 23:

$$
J_{i, \mathrm{tot}}=J_{i, \mathrm{dif}}+x_{i} J_{\text {flow }} \quad i=1, \ldots, n c
$$

Using Eq. 23 to eliminate the diffusive fluxes in Eqs. 22 results in Eqs. 22 again for the total molar fluxes as the terms containing the molar flux caused by flow exactly cancel.

As Eqs. 22 predict molar fluxes relative to each other, only $n c-1$ independent equations can be obtained for a system containing $n c$ species, and therefore Eqs. 22 applied to both $z=0$ and $z=L$ give only eight equations. Therefore, still two additional equations are required to solve the molar fluxes $J_{i, b-i}$, one at $z=0$ and the other at $z=L$, specifying either an additional relation between the fluxes or specifying one of the molar fluxes. By these additional equations the molar fluxes are given relative to a fixed position, that is, the membrane. The two mass balances at the membrane interfaces that seemed to be superfluous owing to the number of boundary conditions at the membrane interfaces required, turn out to be necessary as fixing equations. Therefore, Eqs. $18-20$ are used as boundary conditions at the membrane interfaces at $z=0$ and $z=L$, and Eqs. 21 are used as fixing equations at $z=0$ and $z=L$ for Eqs. 22.

Because Eqs. 22 introduce new derivatives, eight additional boundary conditions are required to solve Eqs. 22. Boundary conditions used with Eqs. 22 are four mole fractions $x_{A \text {,bulk, }}$, $x_{B, \text { bulk }}, x_{C \text {,bulk }}$ and $x_{D, \text { bulk }}$, in the bulk of gas at both sides of the membrane. The pressure $P$ in the bulk of gas at both sides of the membrane must also be specified because of Eq. 17 at $z=0$ and $z=L$. Finally, the mole fraction of inert in the bulk of gas at both sides is determined by the condition that the sum of the mole fractions in the bulk must be equal to 1 (Eq. 9).

\section{Solution Method}

The set of equations (Eqs. 4-22) can be solved only numerically. The numerical method used to solve the equations is a relaxation method (Press et al., 1986). In this relaxation method, the set of $N$ differential equations is replaced by a set of $N^{*} M$ finite difference equations on a grid consisting of $M$ grid points by using a suitable discretization scheme. The discretization of the first- and second-order derivatives of a variable $v$ to the location in the membrane are based on the discretization scheme given in Eq. 24:

$$
\left.\frac{d v}{d z}\right|_{k}=\frac{v_{k+1}-v_{k-1}}{2 h} \text { and }\left.\frac{d^{2} v}{d z^{2}}\right|_{k}=\frac{v_{k+1}-2 v_{k}+v_{k-1}}{h^{2}}
$$

Special attention must be paid to the discretization of the boundary conditions given by Eqs. 18-21. These equations require molar fluxes at the membrane interface which cannot be calculated according to the discretization scheme given by Eq. 24, since discretization uses values of the mole fractions and pressures one grid point backward and one grid point forward, and one of the two is not available at the membrane interfaces. Using an alternative discretization scheme at the interfaces is dangerous because of different truncation errors introduced possibly resulting in numerical convergence without satisfying overall mass balances. As the reaction zone is normally located somewhere inside the membrane and not very close to one of the interfaces, the molar fluxes at the interfaces are replaced by the molar fluxes one grid point into the membrane giving Eqs. 25-28:

$$
\begin{aligned}
\frac{1}{\nu_{B}}\left(J_{B, b-i}-J_{B, k}\right)-\frac{1}{\nu_{A}}\left(J_{A, b-i}-J_{A, k}\right)= & \\
& k=2 \text { and } k=m-1
\end{aligned}
$$

$\frac{1}{\nu_{C}}\left(J_{C, b-i}-J_{C, k}\right)+\frac{1}{\nu_{A}}\left(J_{A, b-i}-J_{A, k}\right)=0$

$$
k=2 \text { and } k=m-1
$$

$$
\begin{aligned}
& \frac{1}{\nu_{D}}\left(J_{D, b-i}-J_{D, k}\right)+\frac{1}{\nu_{A}}\left(J_{A, b-i}-J_{A, k}\right)=0 \\
& k=2 \text { and } k=m-1 \\
& J_{\text {inert }, b-i}=J_{\text {inert }, k} \quad k=2 \text { and } k=m-1
\end{aligned}
$$

Although physically it is possible that the reaction zone becomes located entirely at the membrane interface this will result in severe transport of reactant to the opposing side. The model is not able to predict correct molar fluxes in this case. The numerical technique produces a matrix equation: 


$$
\left[\begin{array}{ccc}
\frac{\partial E_{1, k=1}}{\partial v_{1, k=1}} & \cdots & \frac{\partial E_{1, k=1}}{\partial v_{N, k=M}} \\
\cdot & & \cdot \\
\cdot & \cdot \\
\cdot & & \\
\cdot & \cdot \\
\partial E_{N, k=M} & \cdots & \frac{\partial E_{N, k=M}}{\partial v_{N, k=1}}
\end{array}\right] *\left[\begin{array}{c}
d v_{1, k=1} \\
\cdot \\
d v_{N, k=1} \\
d v_{1, k=2} \\
\cdot \\
\cdot \\
d v_{N, k=M}
\end{array}\right]=\left[\begin{array}{c}
d E_{1, k=1} \\
\cdot \\
d E_{N, k=1} \\
d E_{1, k=2} \\
\cdot \\
\cdot \\
d E_{N, k=M}
\end{array}\right]
$$

with $E_{i, j}$ the deviation of equation number $i$ on grid point number $j$.

$$
d v_{i, j}=v_{i, j \text { old }}-v_{i, \text { new }}
$$

where $v_{i, j \text { old }}$ is the present value of variable $v_{i}$ at $k=j$

$$
d E_{i, j}=E_{i, j \text { old }}-E_{i, j \text { new }}=E_{i, j \text { old }}
$$

A Newton Raphson iteration was used to solve Eq. 29, and in the present study analytical expressions were used for the partial derivatives of the $N^{*} M$ equations with respect to all $N^{*} M$ variables.

Another point requiring attention is the calculation of the molar fluxes from the bulk of the gas to the membrane $J_{i, b-i}$, which should be obtained by solving the set of differential equations (Eqs. 22). Instead of solving these differential equations (Eqs. 22), they are discretized giving:

$$
\sum_{j=1}^{j=n c} \frac{\bar{x}_{j} J_{i, b-i}-\bar{x}_{i} J_{j, b-i}}{D_{i j}^{o}}=-\frac{P}{R T} \frac{x_{i, \text { int }}-x_{i, \text { bulk }}}{\delta}
$$

with

$$
\bar{x}_{i}=\frac{x_{i, \text { bulk }}+x_{i, \text { int }}}{2}
$$

It can be calculated that the deviation of the molar fluxes obtained from the linearized equations compared to the molar fluxes obtained from solving the differential equations is always less than $2 \%$, provided that the system is very diluted and the molar masses of the components do not differ much.

Simulations were performed on a AT-compatible computer provided with a mathematical coprocessor. The number of grid points used varied between 50 and 300 depending on the situation simulated. The number of grid points used was increased until overall mass balances were satisfied within $0.5 \%$.

\section{Numerical Verification}

For a number of asymptotic cases of the model developed in the present study, analytical or approximate analytical solutions can be derived. Therefore, it is possible to compare the results of the simulations of the numerically solved model with those of the analytical or approximate analytical solutions. In this way, a verification of the model is obtained. The asymptotic cases studied are an instantaneous, irreversible reaction in the absence and in the presence of a pressure difference over the membrane. Different situations are being studied using the Claus reaction as a model reaction (Eq. 1) (Mark et al., 1983):

$$
2 \mathrm{H}_{2} \mathrm{~S}+\mathrm{SO}_{2} \leftrightarrow \frac{3}{8} \mathrm{~S}_{8}+2 \mathrm{H}_{2} \mathrm{O}
$$

Data concerning the Claus equilibrium are offered by Gamson and Elkins (1953) and by Verver (1984), see Table 1. The analytical solutions of the asymptotic cases are derived using the Fick diffusion equation to describe multicomponent diffusion, which is allowed only in the case of very diluted, pseudobinary systems, and therefore the mole fractions of $\mathrm{H}_{2} \mathrm{~S}, \mathrm{SO}_{2}$, $\mathrm{H}_{2} \mathrm{O}$ and $\mathrm{S}_{8}$ in the bulk of the gas phase at both sides of the membrane are fixed at low values (equal to or less than 0.01 ), the balance being $\mathrm{N}_{2}$. Surface diffusion has been omitted in these simulations by setting the adsorption parameters $b_{i}$ equal to zero.

\section{Instantaneous, irreversible reaction in the absence of a pressure difference over the membrane}

In the case of an instantaneous, irreversible reaction in the absence of a pressure difference over the membrane, analytical expressions for the molar fluxes can easily be derived (see Sloot, 1991). Mass-transfer resistances in the gas phase outside the membrane were neglected.

The molar fluxes calculated by the numerical model agree well with the analytically obtained molar fluxes. The numerically calculated molar fluxes are slightly lower $( \pm 0.3 \%)$, which could be caused by the fact that in contrast to what is assumed in the analytical solution, the Claus reaction is not entirely irreversible at the conditions used in the calculations (Sloot et al., 1990).

\section{Instantaneous, irreversible reaction in the presence of a} pressure difference over the membrane

It is possible to derive an approximate analytical solution of the molar flux of $\mathrm{H}_{2} \mathrm{~S}$ into the membrane as a function of the pressure difference over the membrane in the situation of very diluted systems where multicomponent diffusion can be described by Fick's law. It is necessary to assume that the reaction is irreversible and the pressure difference over the membrane must be low compared to the pressure level to use constant effective diffusion coefficients that are independent of pressure. For the derivation of this approximation solution the reader is referred to Sloot (1991). From the comparison, it can be concluded that the agreement between the numerically and analytically calculated molar fluxes of $\mathrm{H}_{2} \mathrm{~S}$ into the mem-

Table 1. Parameters Used in the Simulations

Membrane parameters: $\begin{aligned} L & =3 \times 10^{-3} \mathrm{~m} \\ \epsilon / \tau & =0.10 \\ d_{p} & =1 \mu \mathrm{m}\end{aligned}$

Adsorption parameters: $b_{i}=0 \mathrm{~Pa}^{-1}\left(i=\mathrm{H}_{2} \mathrm{~S}, \mathrm{SO}_{2}, \mathrm{~S}_{8}, \mathrm{H}_{2} \mathrm{O}\right.$, and $\left.\mathrm{N}_{2}\right)$ Gas composition:

\begin{tabular}{c|c|c|c|c|c} 
& $x_{\mathrm{H}_{2} \mathrm{~S}}$ & $x_{\mathrm{SO}_{2}}$ & $x_{\mathrm{S}_{8}}$ & $x_{\mathrm{H}_{2} \mathrm{O}}$ & $P$ (bara) \\
\hline $\mathrm{H}_{2} \mathrm{~S}$ side: & $2 \times 10^{-3}$ & $1 \times 10^{-15}$ & $1 \times 10^{-4}$ & $2 \times 10^{-3}$ & 1.0 \\
$\mathrm{SO}_{2}$ side: & $1 \times 10^{-15}$ & $2 \times 10^{-3}$ & $1 \times 10^{-4}$ & $2 \times 10^{-3}$ & 1.0
\end{tabular}

Temperature: $T=473 \mathrm{~K}$

Equilibrium constant:

$$
K_{\text {eq }}=0.924 \times 10^{-6} \exp \left(1.274 \times 10^{4} / T\right)\left(10^{5} \mathrm{~Pa}\right)(\text { Verver, 1984) }
$$

Number of grid points: 300 

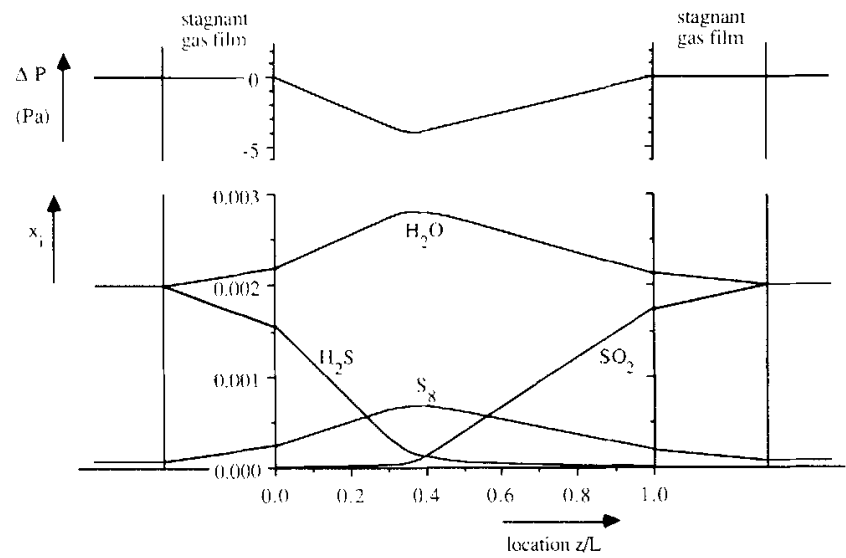

Figure 1. Profiles in the absence of surface diffusion and without a pressure difference at a temperature of $473 \mathrm{~K}$.

brane is satisfactory with respect to the approximations necessary for the calculation of molar fluxes according to the approximate analytical solution. The numerically calculated molar fluxes are slightly lower $( \pm 7 \%)$.

\section{Numerical Evaluation of the Transport Model}

First, simulations were carried out for conditions without surface diffusion both in the absence and in the presence of a pressure difference over the membrane. Next simulations study the influence of surface diffusion both in the absence and in the presence of the instantaneous reversible reaction. In the latter simulations, no pressure difference over the membrane was present.

\section{Simulations in the absence of a pressure difference over the membrane}

Simulations were performed at a room temperature of 473 $\mathrm{K}$ in the absence of surface diffusion. In Figure 1, the mole fraction profiles and the pressure profile inside the membrane are presented. Parameters used in this simulation are given in Table 1. Figure 1 shows that at $473 \mathrm{~K}$ the reaction zone, owing to the high equilibrium constant of the Claus reaction, is small, and at these conditions and this temperature the Claus reaction can be considered to be almost irreversible. As can be seen from the decrease in the mole fraction of the reactants $\mathrm{H}_{2} \mathrm{~S}$ and $\mathrm{SO}_{2}$, and the increase in the mole fraction of the products $\mathrm{S}_{8}$ and $\mathrm{H}_{2} \mathrm{O}$ from the bulk of the gas to the membrane interface, mass-transfer resistances in the gas phase are important for the conditions specified in Table 1 and must be taken into account.

The pressure inside the membrane shows a minimum at the location of the reaction zone although there is no overall pressure difference over the membrane. The occurrence of this minimum can be attributed to the fact that the number of gas molecules is reduced by the chemical reaction (see Eq. 1). Moreover, this net sink in molecules is sufficiently strong to cause a reduced pressure and to induce a viscous flow. As a side effect, bulk flow of inert specie toward the reaction zone is induced, which requires an equal to and opposite diffusive flux of inert to maintain a mass balance.
Table 2. Conditions Used in the Simulations of Surface Diffusion of $\mathrm{H}_{2} \mathrm{~S}$ through the Membrane*

Gas composition:

\begin{tabular}{r|c|c|c|c|c} 
& $x_{\mathrm{H}_{2} \mathrm{~S}}$ & $x_{\mathrm{SO}_{2}}$ & $x_{\mathrm{S}_{\mathrm{x}}}$ & $x_{\mathrm{H}_{2} \mathrm{O}}$ & $P$ (bara) \\
\hdashline$\ldots \ldots$ & $* *$ & $1 \times 10^{-8}$ & $1 \times 10^{-2}$ & 1.0 \\
$\mathrm{H}_{2} \mathrm{~S}$ side: & $1 \times 10^{-2}$ & $* *$ & $1 \times 10^{-8}$ & $1 \times 10^{-4}$ & 1.0 \\
$\mathrm{SO}_{2}$ side: & $1 \times 10^{-5}$ & $* *$ & $1 \times 10^{-4}$
\end{tabular}

(Sloot et al., 1991)

* Other additional parameters are given in Table 1.

** Equilibrium fraction according to $\mathrm{Eq} .35$.

Figure 2 shows dependence of the molar flux of $\mathrm{H}_{2} \mathrm{~S}$ and of the created pressure difference on the pore diameter of the membrane. As a change of the pore diameter affects the masstransfer resistance in the membrane, this also results in a change of the contribution of the mass-transfer resistances in the gas phase outside the membrane. Therefore, these simulations were performed with negligible mass-transfer resistances in the gas phase outside the membrane to eliminate this effect. Since the mass-transfer resistance in the gas phase is eliminated and the reaction is assumed to be instantaneous compared to the masstransfer rate at the membrane interfaces, the composition of the gas at both sides of the membrane must be set at equilibrium in these simulations. Otherwise, a considerable conversion would occur at the interfaces of the gas phase and the membrane.

For small pore diameters $\left(<10^{-8} \mathrm{~m}\right)$ transport takes place in the Knudsen regime, and as the Knudsen diffusion coefficient is directly proportional to the pore diameter (Eq. 17), the molar flux of $\mathrm{H}_{2} \mathrm{~S}$ is directly proportional to the pore diameter. The mole fraction gradient of inert in the membrane is independent of the pore diameter, because the transport of all components is affected identically by the pore diameter. Therefore, the difference between the fraction of inerts in the bulk of the gas and at the reaction zone remains constant. As for small pores, the transport of inert caused by a pressure gradient is determined by Knudsen diffusion and not by viscous flow. Both the diffusion due to a mole fraction gradient of inert and the transport due to a pressure gradient depend in the same way on the pore diameter of the membrane. Therefore, the pressure minimum developed inside the membrane

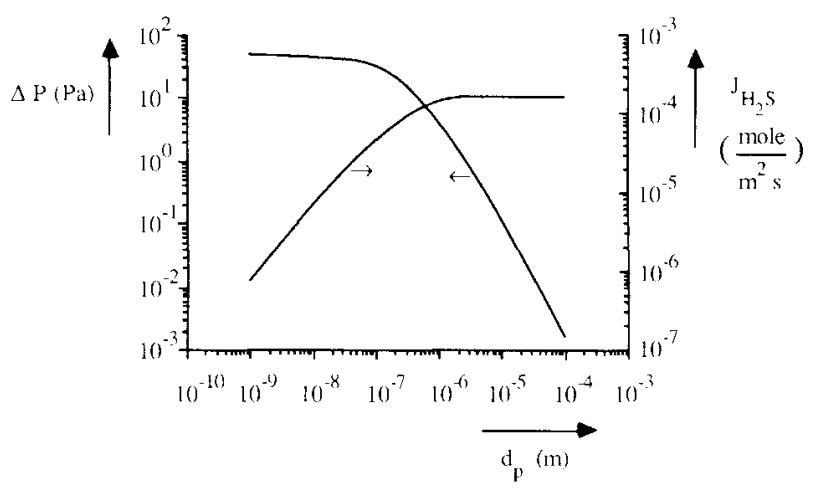

Figure 2. Molar flux of $\mathrm{H}_{2} \mathrm{~S}$ and $\Delta P$ at the pressure minimum as a function of the pore diameter. 
for a small pore diameter is independent of the pore diameter, as can be seen in Figure 2.

For large pore diameters $\left(>10^{-6} \mathrm{~m}\right)$, transport takes place in the continuum regime and the molar flux of $\mathrm{H}_{2} \mathrm{~S}$ is not influenced by the pore diameter because the continuum diffusion coefficient is independent of the pore diameter of the membrane. Also for large pores, the difference between the fraction of inert in the bulk of the gas and at the reaction zone remains constant. Therefore, the transport of inert due to diffusion is also independent of the pore diameter. The transport by viscous flow of inert into the membrane due to a pressure minimum, induced by the net change of molecules due to the chemical reaction, increases with $d_{p}^{2}$ (Eqs. 15 and 18). To maintain a mass balance for the inert, the pressure difference being developed must be proportional to $d_{p}^{-2}$, as shown in Figure 2.

Figure 2 also shows that the pore diameter should be at least $1 \mu \mathrm{m}$ by preference to obtain high molar fluxes. For larger pore diameters, the molar fluxes remain constant. However, the increase in the amount of viscous flow of inert in the case of a pressure difference over the membrane occurs (Sloot et al., 1990) with larger pore diameter.

\section{Numerical simulations in the presence of a pressure dif- ference over the membrane}

The membrane reactor usually is operated without a pressure difference over the membrane as the reactants must diffuse in stoichiometric ratio from opposite sides into the membrane. In practice, a pressure difference over the membrane can occur, for example, in the case of different pressure drops along the membrane area on both sides of the membrane. Therefore, it is necessary to determine the influence of a pressure difference over the membrane on the reactor performance. These effects have been extensively explained (Sloot et al., 1990), and from the simulations with the extended model it can be concluded that the occurrence of a pressure difference over the membrane results in similar phenomena as those observed with the simple model (see Sloot et al., 1990).

\section{Numerical simulations in the presence of surface dif- fusion and in the absence of a chemical reaction}

The transport in the membrane, owing to the combination of diffusion through the gas phase in the pores and surface diffusion, was studied in the absence of a chemical reaction. As was demonstrated by the simulations with a small reaction zone inside the membrane, the mole fractions of $\mathrm{H}_{2} \mathrm{~S}$ and $\mathrm{SO}_{2}$ are very low at the location of the reaction zone. It is especially interesting to study the behavior of surface diffusion at these low mole fractions. In the simulations, mass-transfer resist-

Table 3. Surface Diffusion Parameters of $\mathrm{H}_{2} \mathrm{~S}$ and $\mathrm{SO}_{2}$

\begin{tabular}{cccc}
\hline & $T(\mathrm{~K})$ & $b_{i}\left(\mathrm{~Pa}^{-1}\right)$ & $D_{i, S} c_{S} b_{i}(\mathrm{~mol} / \mathrm{Pa} \cdot \mathrm{s})$ \\
\hline $\mathrm{H}_{2} \mathrm{~S}$ & 484 & $1.25 \times 10^{-3}$ & $1.28 \times 10^{-17}$ \\
& 521 & $1.00 \times 10^{-3}$ & $1.60 \times 10^{-17}$ \\
& 557 & $5.1 \times 10^{-4}$ & $9.4 \times 10^{-18}$ \\
$\mathrm{SO}_{2}$ & 498 & $1.1 \times 10^{-4}$ & $7.4 \times 10^{-18}$ \\
& 525 & $1.36 \times 10^{-3}$ & $1.50 \times 10^{-17}$ \\
& 548 & $9.0 \times 10^{-4}$ & $1.31 \times 10^{-17}$ \\
\hline
\end{tabular}

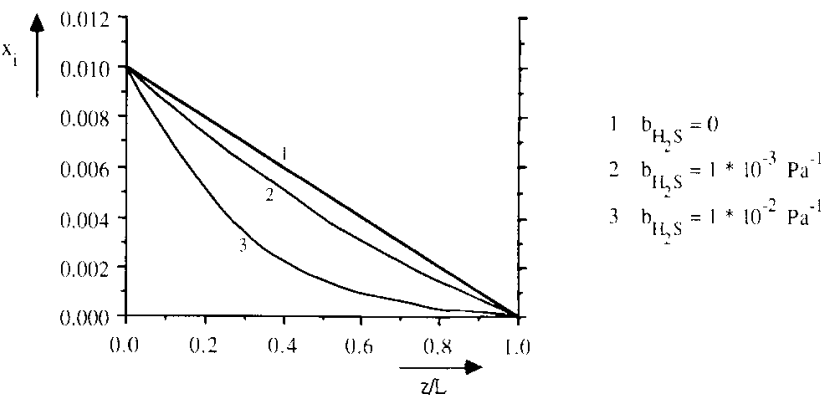

Figure 3. Molar fraction profiles of $\mathrm{H}_{2} \mathrm{~S}$ for different val. ues of the adsorption parameter $b_{\mathrm{H}_{2} \mathrm{~S}}$ in the absence of a chemical reaction.

ances in the gas phase outside the membrane were neglected. In Table 3 the conditions used in the simulations are specified. Only $\mathrm{H}_{2} \mathrm{~S}$ is assumed to adsorb.

In Figure 3 the mole fraction profiles of $\mathrm{H}_{2} \mathrm{~S}$ in the membrane are presented for different values of the adsorption parameter $b_{\mathrm{H}_{2} \mathrm{~S}}$, and in Figure 4 the corresponding pressure profiles are given. Figure 3 shows that in the absence of surface diffusion, $b_{\mathrm{H}_{2} \mathrm{~S}}=0$ and for low values of the adsorption parameter, $b_{\mathrm{H}_{2} \mathrm{~S}}$, the expected straight mole fraction profile is obtained. However, for increasing values of the adsorption parameter, the profiles become curved and simultaneously a minimum in the pressure is developed. These effects can be explained by the degree of coverage of the surface by $\mathrm{H}_{2} \mathrm{~S}$. At low values of $b_{\mathrm{H}, \mathrm{S}}$ the degree of coverage of the surface is relatively small and the gradient in the degree of coverage with the location is linear resulting in a certain constant molar flux of $\mathrm{H}_{2} \mathrm{~S}$ over the surface besides the transport through the gas phase in the pores of the membrane. Increasing the value of $b_{\mathrm{H}_{2} \mathrm{~S}}$ results in an increasing degree of coverage of the surface, and at the membrane side with the highest mole fraction of $\mathrm{H}_{2} \mathrm{~S}$ the gradient in the degree of coverage is relatively small (Eq. 20). At the side with the lower mole fraction of $\mathrm{H}_{2} \mathrm{~S}$ this gradient is steeper resulting in an increase of the molar flux of $\mathrm{H}_{2} \mathrm{~S}$ owing to surface diffusion. Since the net flux of $\mathrm{H}_{2} \mathrm{~S}$ must be independent of position, this difference in these high and low mole fraction surface fluxes must be accommodated by the molecular diffusive flux and the viscous flow flux. Because of the adsorption equilibrium, the free pore molecular diffusion is not independent of the surface diffusion. For that matter, even the viscous flow is not truly independent. The relatively rapid surface diffusion in the low mole fraction side of the membrane

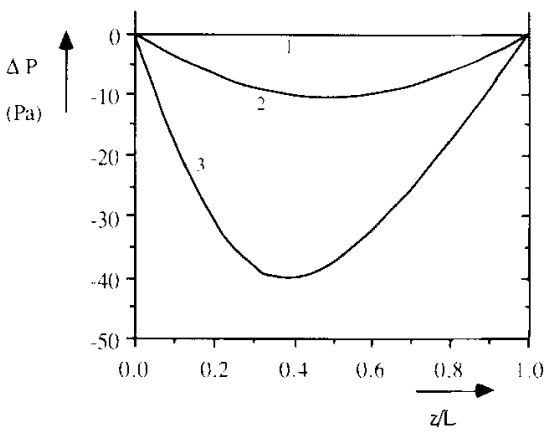

$1 \mathrm{~b}_{\mathrm{H}_{2} \mathrm{~S}}=0$
$2 b_{\mathrm{H}_{2} \mathrm{~S}}=1 * 10^{-3} \mathrm{~Pa}^{-1}$
$3 b_{\mathrm{H}_{2} \mathrm{~S}}=1 * 10^{-2} \mathrm{~Pa}^{-1}$

Figure 4. Pressure profiles inside the membrane belonging to the simulations in Figure 3. 
Table 4. Molar Flux of $\mathrm{H}_{2} \mathrm{~S}$ Caused by Combined Transport through a Membrane with a Pore Diameter of $10 \mathrm{~nm}$ and 1 $\boldsymbol{\mu} \mathrm{m}$ as a Function of the Adsorption Parameter $\boldsymbol{b}_{\mathrm{H}_{2} \mathrm{~S}}$

\begin{tabular}{ccc}
\hline & \multicolumn{2}{c}{$J_{\mathrm{H}_{2} \mathrm{~S}}\left(\mathrm{~mol} / \mathrm{m}^{2} \cdot \mathrm{s}\right)$} \\
\cline { 2 - 3 }$b_{\mathrm{H}_{2} \mathrm{~S}}\left(\mathrm{~Pa}^{-1}\right)$ & $d_{p}=1 \times 10^{-8} \mathrm{~m}$ & $d_{p}=1 \times 10^{-6} \mathrm{~m}$ \\
\hline 0 & $1.46 \times 10^{-5}$ & $2.66 \times 10^{-4}$ \\
$1 \times 10^{-5}$ & $7.24 \times 10^{-4}$ & $2.73 \times 10^{-4}$ \\
$1 \times 10^{-4}$ & $6.52 \times 10^{-3}$ & $3.32 \times 10^{-4}$ \\
$1 \times 10^{-3}$ & $3.58 \times 10^{-2}$ & $6.26 \times 10^{-4}$ \\
$1 \times 10^{-2}$ & $6.49 \times 10^{-2}$ & $9.14 \times 10^{-4}$ \\
\hline
\end{tabular}

(compared to the high mole fraction side) causes the total concentration (pressure) to drop inside the membrane. This low interior pressure results in a viscous flow into the membrane. Asaeda et al. (1981) demonstrated that the existence of these pressure profiles inside the membrane, despite the absence of a pressure difference over the membrane, is caused by the change in surface diffusion along the pores of the membrane.

In Table 4 the molar flux of $\mathrm{H}_{2} \mathrm{~S}$ is given for different values of the adsorption parameter $b_{\mathrm{H}_{2} \mathrm{~S}}$. It can be concluded that surface diffusion can play an important role even in membranes with a large pore diameter provided that the surface concentration is high enough compared to the concentration in the gas phase.

\section{Numerical simulations in the presence of a chemical reaction in the membrane}

A numerical simulation was performed in the presence of a chemical reaction inside the membrane and with an adsorption parameter $b_{i}$ for $\mathrm{H}_{2} \mathrm{~S}$ equal to $1 \times 10^{-3} \mathrm{~Pa}^{-1}$. Mole fractions in the bulk of the gas are the same as used in the simulation presented in Figure 1 and are given in Table 1. Figure 5 shows the mole fraction profiles and the pressure profile in the membrane.

It can be concluded that a maximum in the pressure profile is developed (Figure 5), instead of a minimum (Figure 1), and the location of the reaction zone is shifted substantially toward the $\mathrm{SO}_{2}$ side of the membrane. The shift of the reaction zone is caused by the fact that transport of $\mathrm{H}_{2} \mathrm{~S}$ is increased by surface diffusion in contrast to the transport of $\mathrm{SO}_{2}$. To maintain stoichiometric molar fluxes of $\mathrm{H}_{2} \mathrm{~S}$ and $\mathrm{SO}_{2}$ the location of the reaction zone shifts toward the $\mathrm{SO}_{2}$ side.

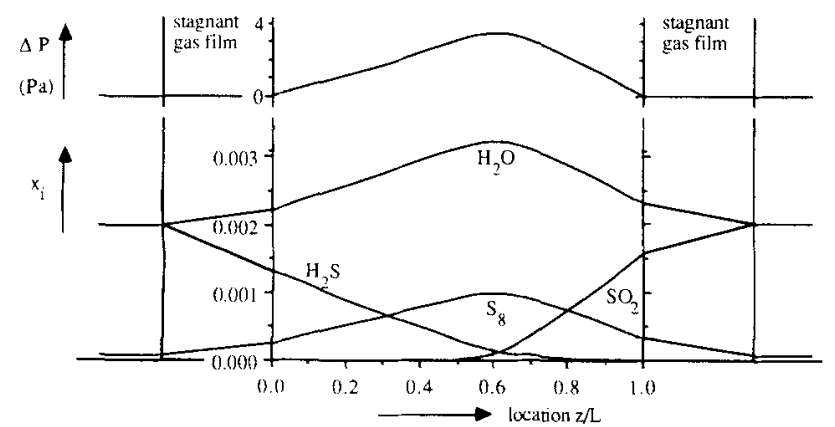

Figure 5. Profiles in the presence of a chemical reaction and surface diffusion without pressure difference over the membrane $\left(b_{\mathrm{H}_{2} \mathrm{~S}}=1 \times 10^{-3} \mathrm{~Pa}^{-1}\right)$.
Due to the increased transport of $\mathrm{H}_{2} \mathrm{~S}$ by surface diffusion, the molar fluxes of the reactants into the membrane are increased, and therefore at the reaction zone a larger amount of products are formed. Consequently, higher molar fluxes of the products out of the membrane are necessary to increase the maximum in the mole fraction profiles of the products at the reaction zone. It appears that the mole fraction of inert at the reaction zone becomes lower than the mole fraction of inert in the bulk of the gas resulting in a driving force for diffusion of inert into the membrane, contrary to the situation in Figure 1. From these simulations it can be concluded that for the determination of whether a maximum or a minimum in the pressure profile is to be expected, it is not enough to look at the stoichiometry of the reaction only (whether the number of gaseous molecules increases or decreases by the reaction), but the mobilities of the different species, the molar flux due to a certain driving force, must also be taken into account.

\section{Numerical Model vs. Previous Model}

A simplified model predicting molar fluxes and mole fraction profiles in the case of an instantaneous reversible reaction inside a membrane with reactants coming from opposite sides of the membrane was presented earlier (Sloot et al., 1990). In this simplified model, transport is described by a combination of viscous flow and molecular diffusion described by Fick's law. By comparing the results of the present model with those obtained from the simplified model, the validity of the approach in the simplified model to describe multicomponent diffusion by Fick's law, not by Stefan Maxwell diffusion relations, can be checked. Situations in the absence and in the presence of a pressure difference over the membrane are studied in this section.

\section{Comparison of both models in the absence of a pressure difference over the membrane}

Simulations were performed at a temperature of $523 \mathrm{~K}$ at different mole fractions of $\mathrm{H}_{2} \mathrm{~S}$ to simulate both in the dilute, pseudo-binary regime where multicomponent diffusion is adequately described by Fick's law and in the concentrated regime where deviations between the models can be expected. In all simulations, the mole fraction of $\mathrm{SO}_{2}$ in the bulk of the gas has been set at 0.75 times the mole fraction of $\mathrm{H}_{2} \mathrm{~S}$ in the bulk of the gas at the $\mathrm{H}_{2} \mathrm{~S}$ side. Mole fractions of the products in the bulk of the gas are fixed at the values in Table 1. The aim of the simulations is to determine whether the present model and the simplified model predict the same molar fluxes. For the conditions of high mole fractions of $\mathrm{H}_{2} \mathrm{~S}$ and $\mathrm{SO}_{2}$ these simulations are not realistic in the sense that the partial pressure of elemental sulfur at the reaction zone inside the membrane exceeds its saturated vapor pressure at $523 \mathrm{~K}$. The ratio of molar fluxes predicted by the simplified model and by the present model is plotted as a function of the mole fraction of $\mathrm{H}_{2} \mathrm{~S}$ in the bulk of the gas at the $\mathrm{H}_{2} \mathrm{~S}$ side. Figure 6 shows the ratio of the molar fluxes of $\mathrm{H}_{2} \mathrm{~S}$ (Figure 6a) of elemental sulfur toward the $\mathrm{H}_{2} \mathrm{~S}$ side (Figure $6 \mathrm{~b}$ ) of the slip (defined as the amount of component $i$ that passes through the membrane to the opposing site) of $\mathrm{SO}_{2}$ and $\mathrm{H}_{2} \mathrm{~S}$ (Figures $6 \mathrm{c}$ and $6 \mathrm{~d}$ ).

In the case of a low mole fraction of $\mathrm{H}_{2} \mathrm{~S}$, both models 

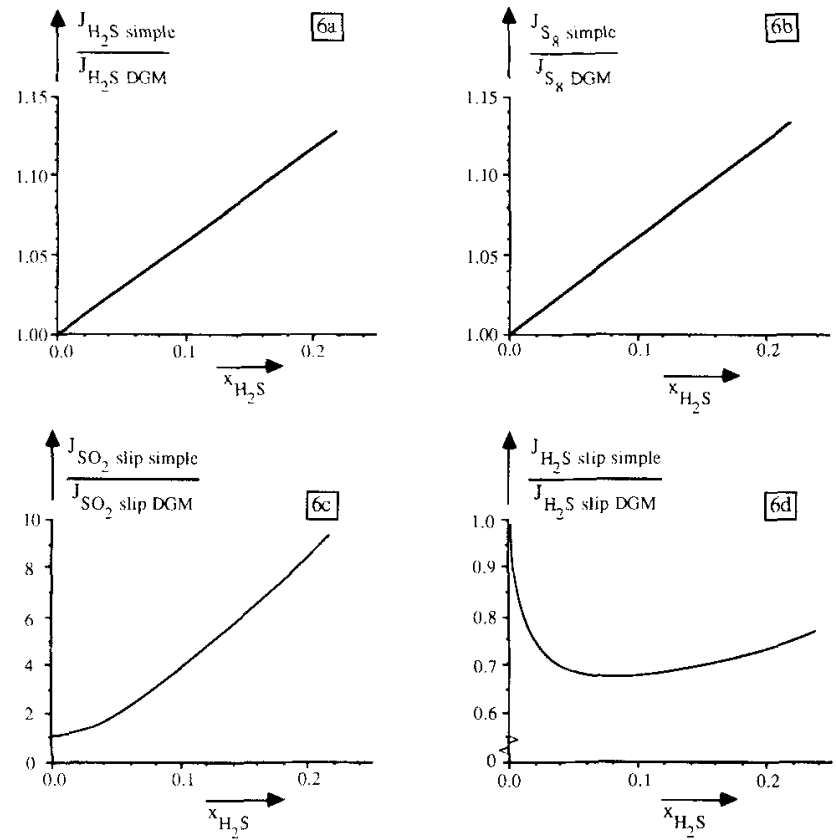

Figure 6. Molar fluxes predicted by transport models in the absence of a pressure difference over the membrane: dusty-gas model vs. simplified model (Sloot et al., 1990).

predict the same molar fluxes of $\mathrm{H}_{2} \mathrm{~S}$ and elemental sulfur toward the $\mathrm{H}_{2} \mathrm{~S}$ side, and the slip of $\mathrm{H}_{2} \mathrm{~S}$ and $\mathrm{SO}_{2}$, Figure 6 . This can be expected in the pseudo-binary regime where multicomponent diffusion can be described adequately by Fick's law. However, the deviation of the molar flux of inert predicted by the models is about $20 \%$. For the inert component, the system cannot be regarded as dilute, and therefore the description of the transport of inert by the simplified model using Fick's law is fundamentally not correct. The difference between the molar fluxes of inert calculated by the different models is probably caused by the nonzero net total molar flux which results in a drag force on the inert component, according to the description of diffusion by Stefan Maxwell. This phenomenon is present in the dusty-gas model, but is not taken into account by the simplified model.

Looking at the ratio of the molar fluxes predicted by both models at higher mole fractions of $\mathrm{H}_{2} \mathrm{~S}$, it appears that the agreement between the molar fluxes of $\mathrm{H}_{2} \mathrm{~S}$ and of elemental sulfur toward the $\mathrm{H}_{2} \mathrm{~S}$ side predicted by both models is reasonably good (15\% difference at maximum). The slip of $\mathrm{H}_{2} \mathrm{~S}$ and $\mathrm{SO}_{2}$ predicted by both models, however, shows large differences, the slip of $\mathrm{H}_{2} \mathrm{~S}$ predicted by the simplified model being lower than that predicted by the present model and the slip of $\mathrm{SO}_{2}$ being higher. These differences can probably be attributed to the fact that the net molar flux is larger than zero. As a consequence, there is a drag force on all components from the $\mathrm{H}_{2} \mathrm{~S}$ side toward the $\mathrm{SO}_{2}$ side, resulting in a small shift of the reaction zone toward the $\mathrm{SO}_{2}$ side. Therefore, the slip predicted by the present model of $\mathrm{H}_{2} \mathrm{~S}$ is larger, and the slip of $\mathrm{SO}_{2}$ is smaller compared to those predicted by the simplified model.

To check these phenomena, a simulation was performed with a reduced mole fraction of $\mathrm{SO}_{2}$ at the $\mathrm{SO}_{2}$ side, the mole fraction of $\mathrm{H}_{2} \mathrm{~S}$ being 0.05 . This caused a net molar flux into the membrane at the $\mathrm{H}_{2} \mathrm{~S}$ side of about zero. As a result, the slip of $\mathrm{SO}_{2}$ predicted by both models deviated less than $0.5 \%$. Surprisingly, the $\mathrm{H}_{2} \mathrm{~S}$ slip predicted by the simplified model is $27 \%$ lower than that calculated by the present model. The net molar flux at the $\mathrm{SO}_{2}$ side is slightly negative, and therefore $\mathrm{SO}_{2}$ is transported into the membrane. In that case it would be expected that the slip of $\mathrm{H}_{2} \mathrm{~S}$ predicted by the present model is lower than that of $\mathrm{H}_{2} \mathrm{~S}$ calculated by the simplified model. Apparently the argument of the net molar flux is a necessary, but not sufficient, condition.

\section{Comparison of models in the presence of a pressure difference over the membrane}

To compare the molar fluxes in the presence of a pressure difference over the membrane calculated by both models, simulations were carried out at a pressure difference of 0.25 bar over the membrane with the highest pressure at the $\mathrm{H}_{2} \mathrm{~S}$ side. Mass-transfer resistances in the gas phase were made negligible. The mole fractions of $\mathrm{H}_{2} \mathrm{~S}$ and $\mathrm{SO}_{2}$ were varied in the same way, and the conditions were kept the same, as described earlier. In Figure 7 the different molar fluxes calculated by both models are compared by giving their ratio as a function of the mole fraction of $\mathrm{H}_{2} \mathrm{~S}$. Although convergence was always obtained by the simplified model, the agreement of the overall mass balances was relatively bad (deviations up to $6 \%$ occurred). Increasing the number of grid points did not reduce these deviations, but the molar fluxes calculated were hardly influenced and are therefore believed to be reliable. In the simulations using the present model this problem did not occur, deviations of the overall mass balances were always less than $0.3 \%$, and the molar fluxes calculated did not change with the increasing number of grid points.
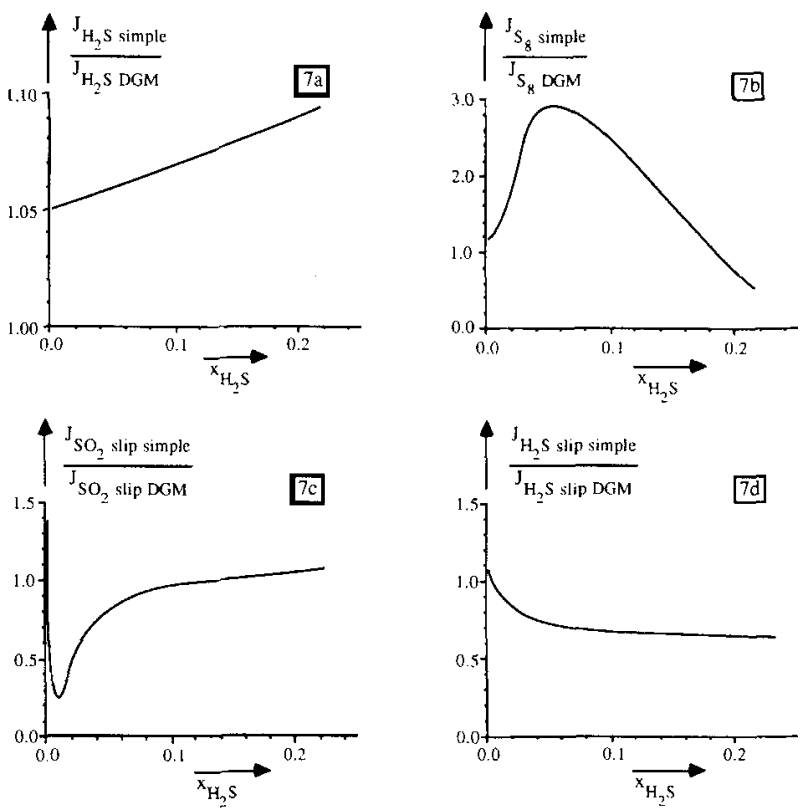

Figure 7. Molar fluxes predicted by transport models in the presence of a pressure difference of 0.25 bar over the membrane: dusty-gas model vs. simplified model (Sloot et al., 1990). 
Figure 7 shows that the molar fluxes of $\mathrm{H}_{2} \mathrm{~S}$ into the membrane predicted by both models agree reasonably, although the molar flux calculated by the simplified model is 5 to $10 \%$ higher than the molar flux calculated by the present model. This is probably caused by the fact that the molar fluxes of inert calculated by the simplified model are always higher resulting in a higher viscous contribution of the molar flux of $\mathrm{H}_{2} \mathrm{~S}$ into the membrane compared to the viscous contribution in the present model.

Large deviation in the molar flux of elemental sulfur toward the $\mathrm{H}_{2} \mathrm{~S}$ side predicted by both models at a mole fraction of $\mathrm{H}_{2} \mathrm{~S}$ of $6 \%$ can be explained in the following way. The molar flux of elemental sulfur toward the $\mathrm{H}_{2} \mathrm{~S}$ side is determined by two oppositely directed contributions; a viscous one resulting in a molar flux of sulfur into the membrane at the $\mathrm{H}_{2} \mathrm{~S}$ side, and a diffusive one resulting in a molar flux out of the membrane to the $\mathrm{H}_{2} \mathrm{~S}$ side. The viscous contribution does not depend very much on the mole fraction of $\mathrm{H}_{2} \mathrm{~S}$ at the conditions studied, but the diffusive contribution depends strongly on the mole fraction of $\mathrm{H}_{2} \mathrm{~S}$ as a higher mole fraction of $\mathrm{H}_{2} \mathrm{~S}$ results in a higher production rate of elemental sulfur inside the membrane, which has to be transported out of the membrane. At mole fractions of $\mathrm{H}_{2} \mathrm{~S}$ up to $1 \%$, the viscous contribution of the transport of elemental sulfur dominates, resulting in an appreciable flux of elemental sulfur into the membrane at the $\mathrm{H}_{2} \mathrm{~S}$ side. At a mole fraction of $\mathrm{H}_{2} \mathrm{~S}$ of $10 \%$ and higher, the diffusive contribution dominates, resulting in an appreciable molar flux of sulfur out of the membrane at the $\mathrm{H}_{2} \mathrm{~S}$ side. In both situations, the deviation between the molar fluxes of sulfur to the $\mathrm{H}_{2} \mathrm{~S}$ side between the models is relatively small. In the case of a mole fraction of $\mathrm{H}_{2} \mathrm{~S}$ between 1 and $10 \%$, the diffusive and the viscous contributions are nearly equal, and therefore the net resulting fluxes of sulfur can be very small. The molar fluxes of inert predicted by the models differ by about $12 \%$ which results in a relatively small difference for both models in the viscous flow. As mentioned previously, the transport of sulfur is due to two approximately compensating transport mechanisms, the relative difference in the total molar fluxes of elemental sulfur to the $\mathrm{H}_{2} \mathrm{~S}$ side predicted by the models can be very pronounced.

The slip of $\mathrm{H}_{2} \mathrm{~S}$ to the $\mathrm{SO}_{2}$ side and vice versa, predicted by the models, are of the same order of magnitude although the differences are up to about $70 \%$. It is remarkable that the agreement in the slip of $\mathrm{SO}_{2}$ calculated by the models is much better than the situation without a pressure difference over the membrane.

\section{Experimental Verification of the Transport Model with the Claus Reaction}

The membrane reactor with separated feeding of reactants is studied experimentally with the Claus reaction as a model reaction $(\mathrm{Eq} .1)$ :

$$
2 \mathrm{H}_{2} \mathrm{~S}+\mathrm{SO}_{2} \rightarrow \frac{3}{8} \mathrm{~S}_{8}+2 \mathrm{H}_{2} \mathrm{O}
$$

In this section, the membrane reactor is studied experimentally, and their molar fluxes are compared with those predicted by the mathematical model using parameters that have been measured independently (Sloot, 1991).

\section{Theory}

When the above mentioned Claus reaction is assumed to be instantaneous and irreversible, the derivation of the molar flux of $\mathrm{H}_{2} \mathrm{~S}$ as a function of the bulk boundary concentrations of $\mathrm{H}_{2} \mathrm{~S}$ and $\mathrm{SO}_{2}$, in the absence of a pressure difference over the membrane, is straightforward:

$$
J_{\mathrm{H}_{2} \mathrm{~S}}=\frac{D_{\mathrm{H}_{2} \mathrm{~S}}^{\mathrm{eff}} c_{\mathrm{H}_{2} \mathrm{~S}, \text { bulk }}+2 D_{\mathrm{SO}_{2}}^{\mathrm{rff}} c_{\mathrm{SO}_{2}, \text { bulk }}}{N}
$$

with

$$
N=L+\frac{D_{\mathrm{H}_{2} \mathrm{~S}}^{\mathrm{eff}}}{D_{\mathrm{H}_{2} \mathrm{~S}}^{o}} \delta_{\mathrm{H}_{2} \mathrm{~S}}+\frac{D_{\mathrm{SO}_{2}}^{\text {cff }}}{D_{\mathrm{SO}_{2}}^{o}} \cdot \delta_{\mathrm{SO}_{2}}
$$

The denominator in Eq. 31 represents three mass-transfer resistances in series; the resistance of the membrane and the resistances in the gas phase outside the membrane at the $\mathrm{H}_{2} \mathrm{~S}$ and $\mathrm{SO}_{2}$ side, respectively. The parameters $\delta_{\mathrm{H}_{2} \mathrm{~S}}$ and $\delta_{\mathrm{SO}_{2}}$ represent the thickness of a stagnant gas film by which the mass transfer resistances outside the membrane are described. The mass-transfer parameter, $k_{G}$, which is equal to the continuum diffusion coefficient divided by the thickness of the stagnant gas film, has been measured experimentally in the membrane reactor as a function of pressure (Sloot, 1991):

$$
k_{G}=\frac{D_{i, \text { inert }}^{o}}{\delta_{i}}=7.53 .10^{-3} \cdot P^{-0.159} \text { with } P \text { in } 10^{5} \mathrm{~Pa}
$$

The effective diffusion coefficients for $\mathrm{H}_{2} \mathrm{~S}$ and $\mathrm{SO}_{2}$ used in Eq. 31 consist of the contributions of both diffusion through the gas phase in the pores of the membrane and surface diffusion. Assuming cylindrically shaped pores, a Langmuir adsorption isotherm and describing surface diffusion by Fick's law, Eq. 33 can be derived for the combination of both transport mechanisms (Sloot, 1991):

$$
D_{i}^{\mathrm{eff}}=D_{i, \mathrm{gas}}^{\mathrm{eff}}+\frac{4}{d_{p}} \frac{D_{i, S}^{\mathrm{erf}} c_{S} b_{i} R T}{\left(1+b_{i} P x_{i, \mathrm{in} \mathrm{O}}\right)\left(1+b_{i} P x_{i, \mathrm{nt} L}\right)}
$$

with

$$
\frac{1}{D_{i, \mathrm{gas}}^{\text {eff }}}=\frac{1}{\frac{\epsilon}{\tau} D_{i, K}^{o}}+\frac{1}{\frac{\epsilon}{\tau} D_{i, \text { inert }}^{o}}
$$

In Eq. $33 b_{i}$ is the adsorption parameter $\left(\mathrm{Pa}^{-1}\right)$, and $c_{S}$ is the concentration of adsorption sites on the surface $\left(\mathrm{mol} / \mathrm{m}^{2}\right)$. The surface diffusion parameters $D_{i, S} c_{S} b_{i}$ and the adsorption parameter $b_{i}$ have been measured independently (Sloot, 1991) as a function of temperature and are given in Table 1.

The mathematical model predicts the molar fluxes as a function of the gas composition at both sides of the membrane using parameters that are measured independently, and therefore no parameters to be fitted remain. These calculated molar fluxes are compared to the experimentally determined molar fluxes here.

Equation 31 is rewritten as Eq. 34 by dividing by the mole fraction of $\mathrm{H}_{2} \mathrm{~S}$ in the bulk of the gas: 


$$
\frac{J_{\mathrm{H}_{2} \mathrm{~S}}}{x_{\mathrm{H}_{2} \mathrm{~S}, \text { bulk }}}=\frac{P}{R T} \cdot \frac{D_{\mathrm{H}_{2} \mathrm{~S}}^{\mathrm{eff}}}{N}+\frac{P}{R T} \cdot \frac{2 D_{\mathrm{SO}_{2}}^{\mathrm{eff}}}{N} \cdot\left(\frac{x_{\mathrm{SO}_{2}, \text { bulk }}}{x_{\mathrm{H}_{2} \mathrm{~S} \text {, bulk }}}\right)
$$

A graphical representation of the experimental data is possible by plotting $J_{\mathrm{H}_{2} \mathrm{~S}} / x_{\mathrm{H}_{2} \mathrm{~S}}$ as a function of $x_{\mathrm{SO}_{2}} / x_{\mathrm{H}_{2} \mathrm{~S}}$. The advantage of this way of plotting (Eq. 34) is that this equation contains only two variables, the molar flux of $\mathrm{H}_{2} \mathrm{~S}$ divided by the mole fraction of $\mathrm{H}_{2} \mathrm{~S}$ and the ratio of the mole fractions of $\mathrm{SO}_{2}$ and $\mathrm{H}_{2} \mathrm{~S}$. From the mole fractions of $\mathrm{H}_{2} \mathrm{~S}$ and $\mathrm{SO}_{2}$ in the bulk of the gas, the molar flux of $\mathrm{H}_{2} \mathrm{~S}$ is calculated from the transport model, and the results can be plotted in the same graph as the experimental data for comparison.

\section{Experimental setup}

The experimental setup used is given in Figure 8. Hydrogen sulfide in nitrogen and sulfur dioxide in nitrogen, with mole fractions of $\mathrm{H}_{2} \mathrm{~S}$ and $\mathrm{SO}_{2}$ of about $1,500 \mathrm{ppm}$, are fed to the membrane reactor by mass-flow controllers. The outlet of the reactor at the $\mathrm{SO}_{2}$ side of the membrane contains a manometer and an additional mass-flow controller. This mass-flow controller is used to adjust the flow out of the reactor at the $\mathrm{SO}_{2}$ side. In this way, the pressure difference over the membrane is fixed at the desired value. Both outlets of the reactor contain a cold trap to condensate the sulfur directly after the reactor to prevent plugging by elemental sulfur produced. The outlet of the reactor at the $\mathrm{H}_{2} \mathrm{~S}$ side of the membrane contains a manometer and a pressure regulator to fix the pressure in the membrane reactor. Both outlets can either be purged or sent to the analyzer to measure conversions of $\mathrm{H}_{2} \mathrm{~S}$ and $\mathrm{SO}_{2}$ simultaneously or determine the slip of either $\mathrm{H}_{2} \mathrm{~S}$ or $\mathrm{SO}_{2}$. In addition, there is the possibility of analyzing the inlets of the reactor, which is not shown in Figure 8 . The pressure difference over the membrane is measured by a U-tube filled with water located at the inlet directly in front of the reactor. The reactor is made of stainless steel and consists of three parts: the $\mathrm{H}_{2} \mathrm{~S}$ chamber, the membrane holder, and the $\mathrm{SO}_{2}$ chamber. The temperature of the reactor is controlled by a Eurotherm. The sealing of the membrane in the holder is described in detail elsewhere (Sloot, 1991). Gases were analyzed by gas chromatography using a FPD detector. The column used was a Chromosil column operated at $323 \mathrm{~K}$.

The membrane used is made of $\alpha-\mathrm{Al}_{2} \mathrm{O}_{3}$ with an average pore diameter of $350 \mathrm{~nm}$ and a thickness of $4.5 \mathrm{~mm}$. The membrane has been impregnated with $0.023 \mathrm{~g} \cdot \mathrm{m}^{-2}$ of a saturated solution of aluminum nitrate, $\mathrm{Al}\left(\mathrm{NO}_{3}\right)_{3}$, and ureum,
$\mathrm{CO}\left(\mathrm{NH}_{2}\right)_{2}$, in water at a temperature of about $373 \mathrm{~K}$. After the impregnated membrane is dried and calcined, $\gamma-\mathrm{Al}_{2} \mathrm{O}_{3}$ results. The porosity-tortuosity factor of the membrane, $\epsilon / \tau$, was determined to be 0.096 (Sloot, 1991).

\section{Experimental results}

Conversion measurements have been performed at temperatures of $493 \mathrm{~K}$ and $542 \mathrm{~K}$ and pressures of 200 and $500 \mathrm{kPa}$. Conversions were determined based on both $\mathrm{H}_{2} \mathrm{~S}$ and $\mathrm{SO}_{2}$. The average conversion of $\mathrm{H}_{2} \mathrm{~S}$ is calculated by:

$$
\zeta_{\mathrm{H}_{2} \mathrm{~S}, \mathrm{av}}=0.5 . \zeta_{\mathrm{H}_{2} \mathrm{~S}}+\zeta_{\mathrm{SO}_{2}}
$$

The molar flux of $\mathrm{H}_{2} \mathrm{~S}$ used in the figures follows from the average conversion of $\mathrm{H}_{2} \mathrm{~S}$ as calculated from Eq. 35 by dividing by the membrane area.

In Figure 9, the results from the conversion measurements at a temperature of $493 \mathrm{~K}$ and a pressure of $220 \mathrm{kPa}$ are presented. In this figure, the theoretically predicted results are also shown. It must be remembered that the calculation of the theoretically predicted molar flux of $\mathrm{H}_{2} \mathrm{~S}$ involves no fitted parameters, but only parameters that have been measured independently.

To check whether the membrane is homogeneous, two series of experiments were performed; the second time using reversed feed, so the $\mathrm{H}_{2} \mathrm{~S}$ is fed to the $\mathrm{SO}_{2}$ side of the membrane and vice versa, and compared to the first series of experiments. This check is important as the parameters required in the theoretical prediction of molar fluxes were determined using either flow or diffusion measurements without reaction. In these measurements, the components are transported through the entire membrane whereas in the conversion measurements $\mathrm{H}_{2} \mathrm{~S}$ and $\mathrm{SO}_{2}$ use only part of the membrane before reaction takes place. Figure 9 shows that the assumption of a homogeneous membrane is reasonable and that the catalyst is present through the entire membrane. It is also clear from Figure 9 that the experimentally determined molar flux of $\mathrm{H}_{2} \mathrm{~S}$ divided by the mole fraction of $\mathrm{H}_{2} \mathrm{~S}$ in the bulk of the gas at the $\mathrm{H}_{2} \mathrm{~S}$ side of the membrane is somewhat lower than the theoretically predicted values, especially at high values of the ratio of the mole fractions of $\mathrm{SO}_{2}$ and $\mathrm{H}_{2} \mathrm{~S}$.

The experimental results are systematically lower than those predicted by theory due mainly to two effects: 1 , the theoretical model assumes the reaction to be instantaneous, whereas in

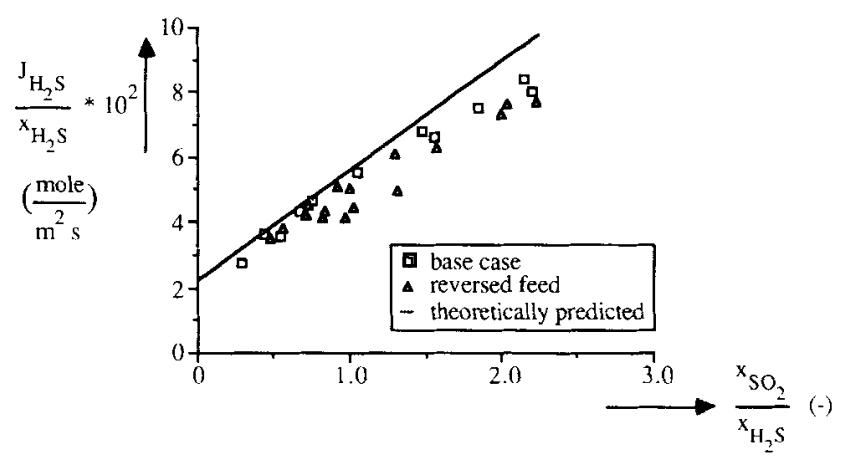

Figure 9. Conversion measurements at a temperature of $493 \mathrm{~K}$ and a pressure of $220 \mathrm{kPa}$.

Figure 8. Experimental setup. 


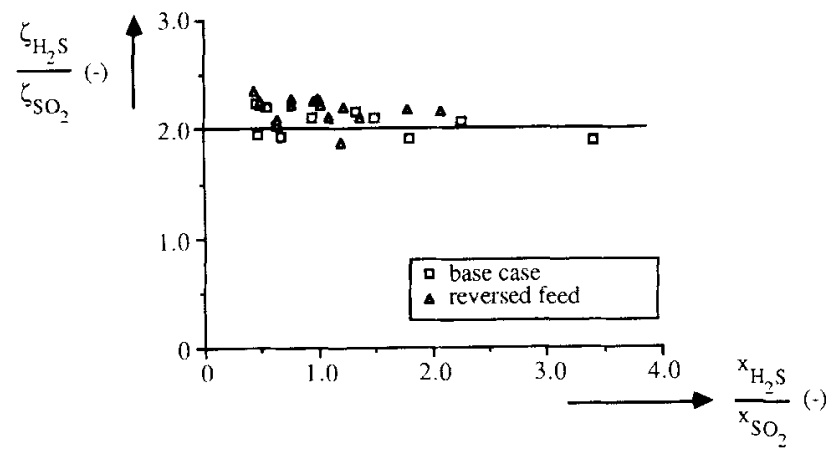

Figure 10. Check of the mass balance at the conversion measurements at $493 \mathrm{~K}$ and a pressure of 220 $\mathrm{kPa}$.

the experiments some influence of kinetics of the Claus reaction might be involved resulting in somewhat lower molar fluxes; 2. the surface diffusion parameters of $\mathrm{H}_{2} \mathrm{~S}$ and $\mathrm{SO}_{2}$ used by the theoretical model could be overestimated. The reaction products, elemental sulfur and water, can possibly adsorb and result in a decrease in the fraction of the adsorption sites available to $\mathrm{H}_{2} \mathrm{~S}$ or $\mathrm{SO}_{2}$. As a result, surface diffusion of $\mathrm{H}_{2} \mathrm{~S}$ and $\mathrm{SO}_{2}$ could be decreased. Determination of the surface diffusion parameters as presented in Table 3 was carried out in the absence of elemental sulfur and water (Sloot, 1991).

In Figure 10, the ratio of the conversions of $\mathrm{H}_{2} \mathrm{~S}$ and $\mathrm{SO}_{2}$ is plotted as a function of the ratio of the mole fractions of $\mathrm{H}_{2} \mathrm{~S}$ and $\mathrm{SO}_{2}$. The agreement between the conversions of $\mathrm{H}_{2} \mathrm{~S}$ and $\mathrm{SO}_{2}$ is reasonable, although the conversion of $\mathrm{H}_{2} \mathrm{~S}$ seems to be systematically higher than that of $\mathrm{SO}_{2}$, especially in the situation with the reversed feed.

To determine whether kinetics play a role in the conversion measurements at $493 \mathrm{~K}$, measurements were also performed at a temperature of $542 \mathrm{~K}$. If kinetics of the Claus reaction influenced the data in Figure 9, the fit of the experimental data by the theoretical model would be expected to be improved at $542 \mathrm{~K}$. To study the influence of surface diffusion, conversion measurements were performed at two pressures: at $220 \mathrm{kPa}$ and at $500 \mathrm{kPa}$. In Figure 11, the molar flux of $\mathrm{H}_{2} \mathrm{~S}$ divided by the mole fraction of $\mathrm{H}_{2} \mathrm{~S}$ is plotted as a function of the ratio of the mole fractions of $\mathrm{SO}_{2}$ and $\mathrm{H}_{2} \mathrm{~S}$.

Figure 11 shows that the experimentally determined values are somewhat lower than those calculated by theory. Again it should be noted that the theoretical model uses only parameters that were determined independently and contains no fitted parameters. The difference between the results measured at pressures of $220 \mathrm{kPa}$ and $500 \mathrm{kPa}$ is remarkable and is caused entirely by the increase in surface diffusion. As the pore diameter of the membrane is $350 \mathrm{~nm}$, transport at a pressure of $220 \mathrm{kPa}$ takes place completely in the continuum regime. As the continuum diffusion coefficient is inversely proportional to the pressure, the molar flux, owing to diffusion through the gas phase in the pores of the membrane, is independent of the pressure. Hence, no difference of the experimental data at $220 \mathrm{kPa}$ and $500 \mathrm{kPa}$ in Figure 11 would be expected due to this cause. The transport due to surface diffusion, however, is linearly proportional to the pressure, provided that the fraction of the adsorption sites covered by $\mathrm{H}_{2} \mathrm{~S}$ or $\mathrm{SO}_{2}$ is small. Therefore, the total molar flux of $\mathrm{H}_{2} \mathrm{~S}$ and $\mathrm{SO}_{2}$ increases with

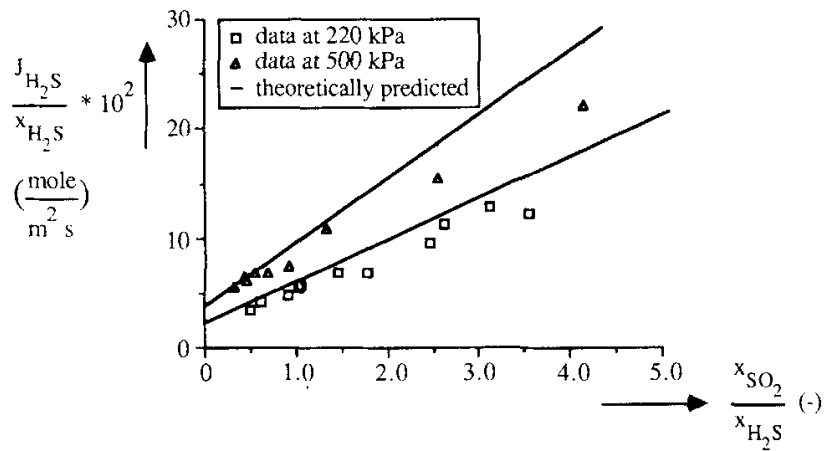

Figure 11. Conversion measurements at a temperature of $542 \mathrm{~K}$ and pressures of 220 and $500 \mathrm{kPa}$.

pressure, as shown in Figure 11. These experimental data demonstrate that despite the large pore diameter of the membrane, surface diffusion causes a considerable part of the total transport.

From the experimental data at a pressure of $500 \mathrm{kPa}$ in Figure 11, it appears that the agreement with the theoretically calculated data is good when the mole fraction of $\mathrm{H}_{2} \mathrm{~S}$ is higher than that of $\mathrm{SO}_{2}$ and worse at relatively high mole fractions of $\mathrm{SO}_{2}$. The most likely explanation for this is that the surface diffusion parameters for $\mathrm{SO}_{2}$, which are important at the pressure of $500 \mathrm{kPa}$, are not correct, although their values have been measured independently (Sloot, 1991).

To check the agreement of the experimentally determined conversions of $\mathrm{H}_{2} \mathrm{~S}$ and $\mathrm{SO}_{2}$, their ratio is plotted as a function of the ratio of the mole fractions of $\mathrm{H}_{2} \mathrm{~S}$ and $\mathrm{SO}_{2}$ in Figures 12 and 13 for a pressure of $220 \mathrm{kPa}$ and $500 \mathrm{kPa}$, respectively. At a pressure of $200 \mathrm{kPa}$, the ratio of the conversion of $\mathrm{H}_{2} \mathrm{~S}$ and $\mathrm{SO}_{2}$ is systematically slightly higher than the stoichiometric ratio of 2 . At a pressure of $500 \mathrm{kPa}$, this ratio is better fulfilled on the average. However, a slight decrease in the conversion ratio with the ratio of the mole fractions of $\mathrm{H}_{2} \mathrm{~S}$ and $\mathrm{SO}_{2}$ is apparent. Nevertheless, the mass balance is fulfilled reasonably in the experiments.

Since a pressure difference over the membrane can occur in practice, it is important to check whether such a pressure difference results in slip of the reactant at the high pressure side of the membrane. Some conversion measurements were carried out in which a pressure difference over the membrane was applied with the highest pressure at the $\mathrm{H}_{2} \mathrm{~S}$ side of the membrane. These measurements were performed at a temperature of $551 \mathrm{~K}$ and an average pressure between 365 and $505 \mathrm{kPa}$. The change in the flows at both sides of the membrane, owing to the pressure difference over the membrane, was fixed by the mass-flow controller in the outlet of the reactor at the $\mathrm{SO}_{2}$ side of the membrane (see Figure 8). In these measurements, no substantial slip of $\mathrm{H}_{2} \mathrm{~S}$ at the $\mathrm{SO}_{2}$ side of the membrane was observed. In Figure 14, the ratio of the experimentally observed molar flux of $\mathrm{H}_{2} \mathrm{~S}$, using Eq. 35, and the theoretically calculated molar flux is plotted as a function of the pressure difference over the membrane.

Figure 14 shows that the agreement between the experimentally observed molar flux and the theoretically calculated molar flux of $\mathrm{H}_{2} \mathrm{~S}$ approaches 1 with higher pressure difference over the membrane. This is probably caused by the fact that at higher pressure differences over the membrane, viscous flow 


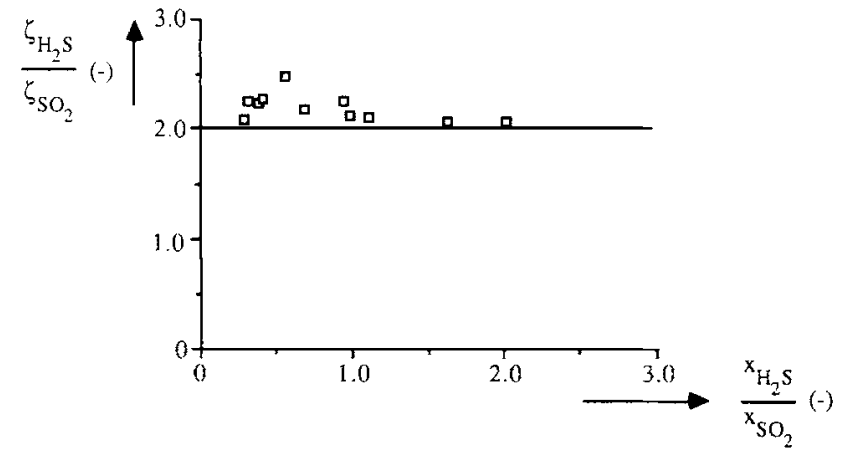

Figure 12. Check of the mass balance for the conversion measurements at $542 \mathrm{~K}$ and $220 \mathrm{kPa}$.

becomes important as a transport mechanism, thereby shifting the reaction zone toward the low pressure side of the membrane, that is, the $\mathrm{SO}_{2}$ side of the membrane. This reduces the relative contribution of surface diffusion of $\mathrm{SO}_{2}$ as a transport mechanism whose parameters are not accurately known under actual conversion conditions.

\section{Conclusions}

The mathematical model presented describes mass transport owing to molecular diffusion and viscous flow, in combination with an instantaneous reversible reaction in a membrane with the reactants coming from opposite sides of the membrane. The model is based on the so-called dusty-gas model (Mason and Malinauskas, 1983) extended with surface diffusion. Masstransfer resistances in the gas phase outside the membrane are taken into account. As a model reaction to study the model of this membrane reactor, the Claus reaction is chosen.

It is demonstrated that pressure profiles inside the membrane are developed even in the absence of an overall pressure difference over the membrane. To determine the occurrence of a maximum or a minimum of the pressure it is necessary to consider the stoichiometry of the reaction as well as the mobilities of the different species.

The present model is used to determine the validity of a previously presented simplified model (Sloot et al., 1990). From this comparison, it is concluded that the simplified model qualitatively predicts the correct molar fluxes including the slip of reactants to the opposite side of the membrane, both in the absence and in the presence of a moderate pressure difference over the membrane, provided that the mole fractions of the

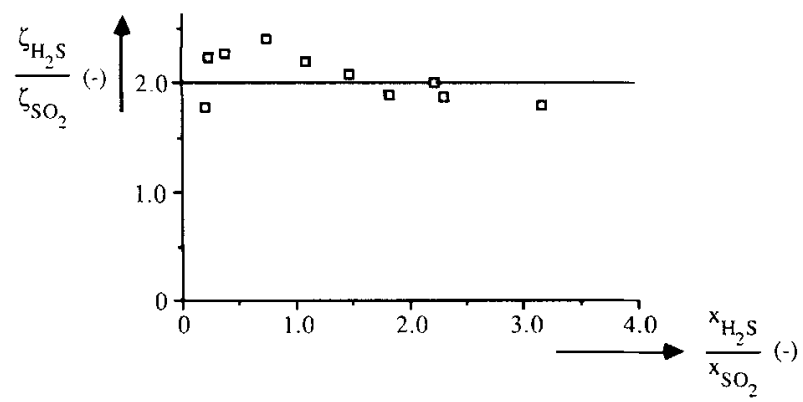

Figure 13. Check of the mass balance for the conversion measurements at $542 \mathrm{~K}$ and $500 \mathrm{kPa}$.

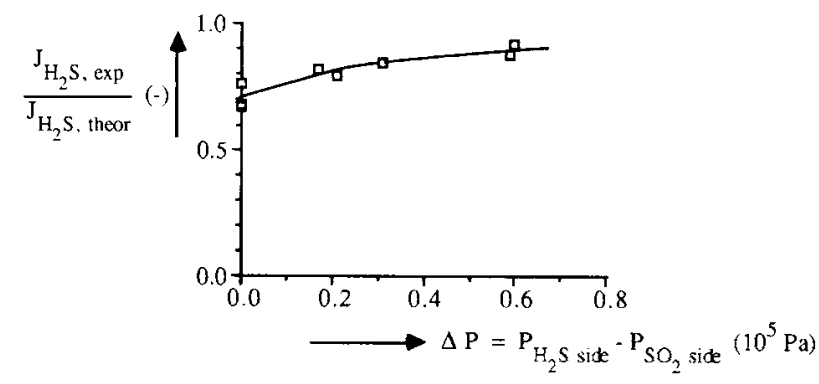

Figure 14. Conversion measurements in the presence of a pressure difference over the membrane.

reactants and products remain lower than those of inert (dilute systems).

The mathematical transport model describing transport and reaction in the membrane reactor is verified experimentally using the Claus reaction as a model reaction. Results from conversion measurements at temperatures of $493 \mathrm{~K}$ and $541 \mathrm{~K}$ are shown to be 10 to $20 \%$ lower than the molar fluxes predicted by the theoretical model. Conversion measurements performed at pressures of $220 \mathrm{kPa}$ and $500 \mathrm{kPa}$ demonstrate the occurrence of surface diffusion as a transport mechanism despite the large pore diameter of the membrane of $350 \mathrm{~nm}$. Measurements in the presence of a pressure difference over the membrane were performed at a temperature of $551 \mathrm{~K}$. Despite the relatively large pressure difference of up to $0.7 \mathrm{bar}$, no substantial slip of $\mathrm{H}_{2} \mathrm{~S}$ to the $\mathrm{SO}_{2}$ side of the membrane was observed. The agreement between the experimentally observed and the theoretically calculated molar flux of $\mathrm{H}_{2} \mathrm{~S}$ was reasonable.

\section{Acknowledgment}

These investigations were supported by the National Dutch Program Committee on Membranes. We acknowledge K. van Bree, W. Leppink, and A. Pleiter for the construction of the experimental setup, as well as $P$. Weersink and $N$. Kuipers for their part in the experimental work.

\section{Notation}

$A_{i j}=$ matrix element defined in Eq. 30

$b_{i}=$ adsorption constant of a component, $\mathrm{Pa}^{-1}$

$B_{o}=$ membrane-specific parameter defined by Eq. $18, \mathrm{~m}^{2}$

$c_{i, \text { bulk }}=$ concentration of component $i$ in the bulk of the gas, $\mathrm{mol} \cdot \mathrm{m}^{-3}$

$c_{S}=$ surface concentration of adsorption sites, $\mathrm{mol} \cdot \mathrm{m}^{-2}$

$D_{i j}=$ effective continuum diffusion coefficient of component $i$ in $j, \mathrm{~m}^{2} \cdot \mathrm{s}^{-1}$

$D_{i K}=$ effective Knudsen diffusion coefficient of component $i$, $\mathrm{m}^{2} \cdot \mathrm{s}^{-1}$

$D_{i s}=$ effective surface diffusion coefficient, $\mathrm{m}^{2} \cdot \mathrm{s}^{-1}$

$D_{i}^{p}=$ continuum diffusion coefficient of component $i, \mathrm{~m}^{2} \cdot \mathrm{s}^{-1}$

$D_{i, \text { eff }}=$ effective diffusion coefficient of component $i, \mathrm{~m}^{2} \cdot \mathrm{s}^{-1}$

$D_{i, K}^{o}=$ Knudsen diffusion coefficient, $\mathrm{m}^{2} \cdot \mathrm{s}^{-}$

$D_{i, \text { seff }}=$ effective surface diffusion coefficient, $\mathrm{m}^{2} \cdot \mathrm{s}^{-1}$

$d_{p}=$ mean pore diameter, $\mathrm{m}$

$E_{i j}=$ deviation of equation $i$ on grid point number $j$

$h=$ membrane thickness $L$ divided by the number of grid points $M, \mathrm{~m}$

$J_{i, \text { gas }}=$ molar flux of component $i$ through the gas phase, $\mathrm{mol} \cdot \mathrm{m}^{-2} \cdot \mathrm{s}^{-1}$

$J_{i, t \text { tot }}=$ total molar flux of component $i, \mathrm{~mol} \cdot \mathrm{m}^{-2} \cdot \mathrm{s}^{-1}$

$J_{i, \text { surf }}=$ molar flux of component $i$ over the surface, $\mathrm{mol} \cdot \mathrm{m}^{-2} \cdot \mathrm{s}^{-1}$

$k=$ grid point number

$k_{G}=$ mass-transfer parameter in the gas phase outside the membrane, $\mathrm{m} \cdot \mathrm{s}^{-1}$ 
$K_{\mathrm{eq}}=$ equilibrium constant

$L=$ membrane thickness, $\mathrm{m}$

$M=$ number of grid points

$M_{i}=$ molar mass, $\mathrm{kg} \cdot \mathrm{mol}^{-1}$

$N=$ parameter defined in $\mathrm{Eq} .32, \mathrm{~m}$

$n c=$ number of components

$P=$ pressure, $\mathrm{Pa}$

$R=$ gas constant, $\mathrm{J} \cdot \mathrm{mol}^{-1} \cdot \mathrm{K}^{-1}$

$R_{i}=$ reaction rate, $\mathrm{mol} \cdot \mathrm{m}^{-3} \cdot \mathrm{s}^{-1}$

$T=$ temperature, $\mathrm{K}$

$v=$ general variable used in Eq. 24

$x=$ mole fraction

$x_{i \text { int }}=$ molar fraction of component $i$ at the membrane interface

$z=$ location inside the membrane, $m$

\section{Greek letters}

$\delta=$ thickness of the stagnant gas film outside the membrane, $m$ $\epsilon=$ porosity of the membrane

$\eta=$ viscosity, $\mathrm{Pa} \cdot \mathrm{s}$

$\nu=$ stoichiometric coefficient

$\theta_{i}=$ fraction of surface covered by component $i$

$\tau=$ tortuosity of the membrane

$\zeta_{i}=$ conversion of component $i, \mathrm{~mol} \cdot \mathrm{s}^{-1}$

\section{Indices}

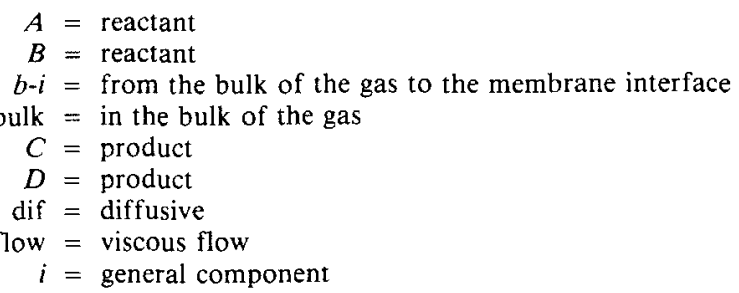

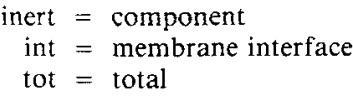

\section{Literature Cited}

Asaeda, M., J. Watanabe, Y. Matono, K. Kojima, and R. Toei, "Combined Surface and Gas-Phase Diffusion through Plugs of Porous Adsorbents in Transition Diffusion Regime," J. Chem. Eng. Jpn., 14(1), 13 (1981).

Gamson, B. W., and R. H. Elkins, "Sulfur from Hydrogen Sulfide," Chem. Eng. Prog., 49(4), 203 (1953).

Kirk-Othmer, Encyclopedia of Chemical Technology, H. F. Mark, D. F. Othmer, C. G. Overberger, and G. T. Seaborg, eds., Vol. 22, Wiley, New York (1983).

Mason, E. A., and A. P. Malinauskas, Gas Transport in Porous Media: the Dusty-Gas Model, Chemical Engineering Monographs 17, Elsevier, Amsterdam (1983).

Press, W. H., B. P. Flannery, S. A. Teukolsky, and W. T. Vetterling, Numerical Recipes: the Art of Scientific Computing, Cambridge Univ. Press, Cambridge (1986).

Reid, R. C., J. M. Prausnitz, and T. K. Sherwood, The Properties of Gases and Liquids, 3rd ed., McGraw-Hill, New York (1977).

Sloot, H. J., G. F. Versteeg, and W. P. M. van Swaaij, "A Nonpermselective Membrane Reactor for Chemical Processes Normally Requiring Strict Stoichiometric Feed Rates of Reactants," Chem. Eng. Sci., 45(8), 2415 (1990).

Sloot, H. J., "A Nonpermselective Membrane Reactor for Catalytic Gas-Phase Reactions," PhD Thesis, Twente Univ. of Technology, The Netherlands (1991).

Verver, A. B,, "The Catalytic Oxidation of Hydrogen Sulphide to Sulphur in a Gas-Solid Trickle-Flow Reactor,"' PhD Thesis, Twente Univ, of Technology, The Netherlands (1984).

Manuscript received Aug. 2, 1991, and revision received Mar. 31, 1992. 